\title{
POLÍTICAS PÚBLICAS DE SAÚDE MENTAL PARA CRIANÇAS E ADOLESCENTES: A ATUAÇÃO DO PSICÓLOGO
}

PUBLIC MENTAL HEALTH POLICIES FOR CHILDREN AND ADOLESCENTS: THE PSYCHOLOGIST'S WORK

POLÍTICAS PÚBLICAS DE SALUD MENTAL PARA NIÑOS Y ADOLESCENTES: LA ACTUACIÓN DEL PSICÓLOGO

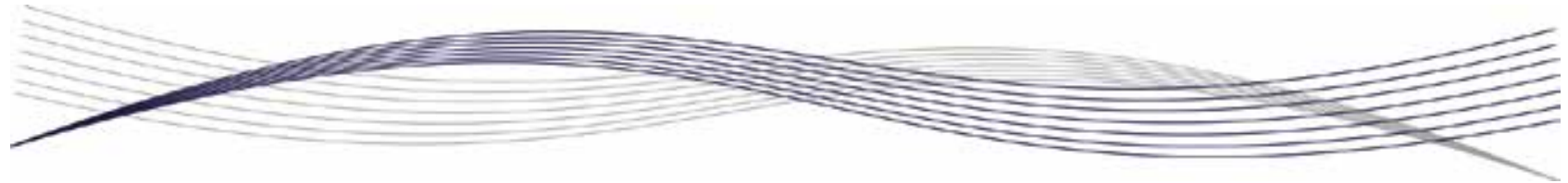

Palavras-chave: Políticas Públicas; Saúde Mental; Criança; Adolescente; Psicólogo.

Keywords:

Public Policy; Mental Health; Child; Adolescent; Psychologist.

Palabras clave: Política Pública; Salud Mental; Niño; Adolescente; Psicólogo.

Submetido: 26/05/2018

Aprovado: 22/11/2018

Autor(a) para Correspondência: Fabiana Cruz Soares R. Aimorés, 190, casa 09 Boa Esperança Parnaíba (PI) CEP: 64215-370 E-mail: fabianacruzsoares@ yahoo.com.br

\section{RESUMO}

Este artigo analisa o processo de desenvolvimento das políticas públicas de saúde mental voltadas ao público infantojuvenil e o papel assumido pelo psicólogo nesse contexto. A metodologia consiste em uma revisão crítica da literatura nacional sobre a saúde mental, realizada nas bases SciELO, Lilacs, PePSIC e Bireme. As políticas de saúde mental para crianças e adolescentes são recentes. As ações anteriores voltadas a esse público se pautavam por institucionalização e isolamento social. Com o advento da Reforma Sanitária e da Reforma Psiquiátrica, novos caminhos de proteção e cuidado foram traçados, proporcionando um novo olhar sobre os jovens. Estes passaram a ser reconhecidos como sujeitos de direitos em caráter de absoluta prioridade. O Estatuto da Criança e do Adolescente (ECA), a Reforma Psiquiátrica e o Sistema Único de Saúde (SUS) reforçaram as disposições da Constituição Federal de 1988 e a Rede de Atenção Psicossocial (RAPS) consolidou uma nova práxis do psicólogo nesse contexto.

\footnotetext{
1. Psicóloga. Mestre em Saúde Coletiva pela Faculdade São Leopoldo Mandic (SLMandic). Professora no Curso de Psicologia do Centro Universitário Maurício de Nassau (UniNassau). Parnaíba (PI), Brasil. E-mail: fabianacruzsoares@yahoo.com.br

2. Aluno de Graduação em Psicologia no UniNassau. Parnaíba (PI), Brasil. E-mail: ramnsesmaster12@gmail.com 3. Psicóloga. Aluna de Pós-Graduação em Tanatologia no Instituto Dexter. Parnaíba (PI), Brasil. E-mail: renata. osc@bol.com.br
} 


\section{ABSTRACT}

This article analyzes the process for developing public mental health policies aimed at the child-juvenile clientele and the role played by the psychologist in this context. The methodology consists of a critical review of the national literature on mental health, carried out on the SCIELO, LILACS, PePSIC, and BIREME databases. The mental health policies for children and adolescents are recent. Previous actions aimed at this clientele were usually based on institutionalization and social isolation. With the advent of the Health Care Reform and the Psychiatric Reform, new protection and health care paths were traced, providing young people with a new look. The latter managed to be recognized as subjects of rights that have absolute priority. The Brazilian Statute of the Child and Adolescent (ECA), the Psychiatric Reform, and the Brazilian National Health System (SUS) reinforced the provisions of the 1988 Brazilian Federal Constitution and the Brazilian Psychosocial Care Network (RAPS) consolidated a new psychologist's praxis in this context.

\section{RESUMEN}

Este artículo analiza el proceso para desarrollar políticas públicas de salud mental dirigidas al público infantojuvenil y el papel jugado por el psicólogo en este contexto. La metodología consiste en una revisión crítica de la literatura nacional acerca de la salud mental, realizada en las bases SCiELO, LILACS, PePSIC y BIREME. Las políticas de salud mental para niños y adolescentes son recientes. Las acciones anteriores dirigidas a este público se basaban generalmente en institucionalización y aislamiento social. Con el advenimiento de la Reforma Sanitaria y la Reforma Psiquiátrica, se trazaron nuevos caminos de protección y cuidado, proporcionando una nueva mirada sobre los jóvenes. Estos últimos lograron ser reconocidos como sujetos de derechos que tienen prioridad absoluta. El Estatuto Brasileño del Niño y del Adolescente (ECA), la Reforma Psiquiátrica y el Sistema Nacional de Salud Brasileño (SUS) reforzaron las disposiciones de la Constitución Federal Brasileña de 1988 y la Red de Atención Psicosocial Brasileña (RAPS) consolidó una nueva praxis del psicólogo en este contexto.

\section{INTRO DUÇÃ 0}

No Brasil, as políticas de saúde mental que priorizam as crianças e os adolescentes são tardias, pois só foram pensadas e planejadas no início do século $X X I^{1}$. Antes desse período, não existiam redes de cuidado voltadas ao público infantojuvenil e esse papel ficava a cargo das áreas da educação e do serviço social. Diante disso, as ações se tornavam mais corretivas e reparadoras do que focadas nas reais necessidades biopsicossociais de cada indivíduo. As crianças e os adolescentes com problemas mentais eram subjugados ao Estado, que pregava uma política de proteção social por meio de institucionalização .

0 modelo de assistência asilar perdurou por muitos anos no país, sob o argumento de que a pobreza era geradora de "crianças abandonadas" e de "jovens delinquentes". Visava a proporcionar maior controle estatal e menor tendimento a necessidades individuais ${ }^{2}$. Nesse contexto, as crianças e os adolescentes com problemas mentais eram qualificados como "incapazes" e o isolamento social era apontado como a "solução mais adequada" para a situação de vulnerabilidade em que se encontravam. À margem dessa situação, também existiam as Santas Casas de Misericórdia, instituições ligadas à Igreja
Católica e destinadas ao recolhimento de crianças abandonadas e de donativos ${ }^{3}$.

A lógica da institucionalização de crianças e adolescentes começou a mudar a partir da criação do Código de Menores, em 1927, também conhecido como Código de Mello Mattos, em alusão ao primeiro juiz de menores da América Latina ${ }^{4}$. Esse foi o primeiro diploma legal voltado ao público infantojuvenil, mas seu foco recaía sobre os "meninos de rua", isto é, as crianças e os adolescentes pobres, reforçando ainda mais o estigma de que eram perigosos e ameaçadores para a sociedade ${ }^{2}$.

A legislação brasileira começou a contemplar a proteção da infância e da adolescência, com disposições contrárias aos castigos físicos e conduzindo a assistência a caminhos mais abertos do que fechados, porém, privilegiava o modelo asilar ${ }^{4}$. 0 Código de Menores foi reformulado em 1979 e já continha o princípio da proteção integral, mas se baseava no paradigma da legislação anterior.

Nesse período, persistiu um claro desinteresse na reinserção social da criança e do adolescente. A legislação se limitava ao controle da ordem pública e da paz social e mostrava-se totalmente desvinculada da visão da criança e do adolescente como sujeitos de direitos ${ }^{5}$. A criança e o adolescente não eram vistos como portadores de sentimentos, 
respeito e consciência, mas como seres humanos moldados ${ }^{6}$. Nesse sentido, o modo diferenciado do mundo infantojuvenil não agradava ao adulto, mas dependia de sua origem e do status que sua condição social representava. Diante dessa realidade, 0 Código de Menores de 1979 se tornou ineficaz. Com a restauração da democracia, o Estatuto da Criança e do Adolescente $(E C A)^{7}$ foi promulgado em 1990 e consiste em um diploma de direitos humanos que contempla o que há de mais avançado nas normas internacionais em relação aos direitos da criança e do adolescente ${ }^{2}$.

Tendo em vista o contexto histórico da saúde mental para crianças e adolescentes no Brasil, constata-se que as atuais políticas de atenção psicossocial constituem conquistas, pois contribuem para enfrentar e superar os desafios envolvidos no cuidado proporcionado a sujeitos de direitos. Assim, este artigo analisa o processo de desenvolvimento das políticas públicas de saúde mental voltadas ao público infantojuvenil e o papel assumido pelo psicólogo nesse contexto.

\section{METODOLOGIA}

Trata-se de revisão crítica da literatura nacional sobre saúde mental, realizada nas bases SciELO, Lilacs, PePSIC e Bireme. Como pesquisa exploratória, descritiva e explicativa, teve por objetivo levantar a produção científica pertinente, articulando os conhecimentos proporcionados por pesquisadores que analisaram a saúde mental em nosso meio ${ }^{8-9}$.

0s critérios de inclusão de estudos foram:

- Publicação no período entre 2012 e 2017;

- Foco temático em políticas públicas voltadas ao público infantojuvenil;

- Abordagem do papel do psicólogo nesse contexto; e

- Pesquisas exclusivamente brasileiras.

Os termos de busca adotados foram "políticas públicas", "saúde mental”, “crianças" e “adolescentes". Com o uso desses descritores, localizamos 19 produções científicas; aplicando os critérios de inclusão estabelecidos, a amostra totalizou 17 publicações a analisar. Esse levantamento bibliográfico ocorreu de abril a junho de 2017, com vistas a investigar os diversos aspectos da construção e reconstrução de políticas públicas de saúde mental voltadas ao público infantojuvenil, relacionando-os à atuação do psicólogo.

Assim, esta revisão de literatura identificou 3 categorias temáticas:

1. Políticas públicas de saúde, Reforma Sanitária e Reforma Psiquiátrica: reflexões e transformações na saúde mental;

2. Políticas de saúde mental para crianças e adolescentes no Brasil; e

3. Atuação do psicólogo em políticas públicas de saúde mental para crianças e adolescentes.

\section{RESULTADOS E DISCUSSÃO}

$0 s$ resultados se organizam em torno das 3 categorias temáticas indicadas na seção anterior.

\section{Políticas Públicas de Saúde, Reforma Sanitária e Reforma Psiquiátrica: reflexões e transformações na saúde mental}

As políticas públicas fazem parte do escopo de análise e ação de diversos profissionais da saúde, entre eles os psicólogos, pois configuram uma complexa rede de inter-relacionamentos. Isso implica a diversidade e subjetividade humana, demandando protagonismo social diante de sentimentos, pensamentos, conflitos e emoções de sujeitos de direitos ${ }^{10}$.

Essa perspectiva humanizada e integral de atenção à saúde é relativamente recente. 0 modelo anterior, denominado biomédico ou hospitalar, centrava-se na cura da doença - e esse era o ideal de saúde vigente no imaginário da sociedade brasileira. A visão atual do setor saúde tem como marco histórico a Reforma Sanitária, ocorrida no Brasil no início da década de 1970, que envolveu todos os profissionais da saúde na busca por melhores condições de vida da população ${ }^{11}$.

0 desenvolvimento dessa nova concepção de saúde decorreu tanto da Reforma Sanitária como da implantação do Sistema Único de Saúde (SUS)

$$
\begin{gathered}
\text {... o Estatuto } \\
\text { da Criança e do } \\
\text { Adolescente [...] e } \\
\text { consiste em um diploma } \\
\text { de direitos humanos... }
\end{gathered}
$$


e encorajou os profissionais da saúde mental a construir sua própria reforma, denominada Reforma Psiquiátrica ${ }^{12}$. Esta busca garantir os direitos dos cidadãos com algum transtorno mental, empoderandoos e reinserindo-os em todos os espaços da sociedade. 0 Movimento dos Trabalhadores em Saúde Mental (MTSM) surgiu em 1978, com propostas de mudanças nas políticas de assistência psiquiátrica vigentes. Vale ressaltar que o MTSM se inspirou em Franco Basaglia, responsável pela Reforma Psiquiátrica italiana, que defendia profundas transformações tanto no modelo de assistência psiquiátrica como na relação da sociedade com a "loucura", tornando o cuidado aos usuários individualizado e humanizado ${ }^{13}$.

Surgiu nesse cenário o Projeto de Lei $n$. 3.657/1989, denominado Lei Paulo Delgado em homenagem ao deputado responsável por sua autoria, que buscava regulamentar os direitos dos indivíduos com transtorno mental e a extinção dos internamentos manicomiais idealizando, assim, uma possivel reforma das políticas de saúde mental ${ }^{14}$. Entretanto, a respectiva lei só foi sancionada em 2001, instituindo uma Reforma Psiquiátrica de fato, na qual os modelos institucionais foram substituídos, a doença mental passou a ser vista de modo mais amplo e a saúde foi redefinida como um fenômeno que envolve todas as esferas biológicas, psicológicas e sociais do ser humano ${ }^{13}$.

0 processo de Reforma Psiquiátrica Brasileira adquiriu aparatos de vital importância no final da década de 1990, com a regulamentação dos centros de atenção psicossocial (CAPS) e o surgimento dos serviços denominados residências terapêuticas. 0s CAPS constituem espaços privilegiados de intervenção para pessoas com transtornos psicológicos graves e persistentes, oferecendo atenção e cuidados clínicos que visam à reabilitação psicossocial. Já as residências terapêuticas são moradias localizadas em espaços urbanos que proporcionam aos usuários suporte e acompanhamento de profissionais especializados, com vistas a promover a desinstitucionalizaçã $0^{14}$. $0 \mathrm{~s}$ trabalhos terapêuticos para a inserção ou reinserção social se mostram extremamente válidos, apesar dos diversos questionamentos a respeito do indivíduo à margem da sociedade; entretanto, além de valorizar as potencialidades e os limites de cada pessoa, estabelecem e/ou fortalecem vínculos que estimulam o protagonismo de cada usuário em relação à sua vida.

Nessa perspectiva, após a III Conferência Nacional de Saúde Mental, realizada no final de 2001,

\section{...vínculos que estimulam o protagonismo de cada usuário em relação à sua vida.}

o Ministério da Saúde normatizou em 2002 o centro de atenção psicossocial infantojuvenil (CAPSi), voltado a crianças e adolescentes com transtornos mentais graves. Nessa modalidade de CAPS, mostrase indispensável o envolvimento da rede de cuidados à saúde e da sociedade como um todo, englobando educação, justiça social e lazer, de modo que a plena atenção à saúde mental da criança e do adolescente se torne uma realidade no país ${ }^{15}$.

\section{Politicas de saúde mental para crianças e adolescentes no Brasil}

A preocupação com a saúde mental da criança e do adolescente assumiu maior destaque com a conversão do Projeto de Lei n. 3.657/1989 na Lei n. 10.216/2001 e a III Conferência Nacional de Saúde Mental foi crucial para levantar novas discussões voltadas a esse público, negligenciado por quase um século. As transformações das políticas públicas que vieram a lume nos anos posteriores colocaram em pauta os direitos das crianças e dos adolescentes com deficiência mental, que devem ter acesso a uma rede de atenção e cuidado à saúde capaz de melhorar sua qualidade de vida ${ }^{16}$.

$0 \mathrm{ECA}^{7}$ reafirma tais direitos por meio do paradigma de proteção integral, reforçando o caráter de prioridade absoluta dos seres humanos em desenvolvimento, sob responsabilidade do Estado em atuação conjunta com a sociedade e as famílias. Nesse sentido, o art. $4^{\circ}$ do ECA dispõe que:

É dever da família, da comunidade, da sociedade em geral e do poder público assegurar, com absoluta prioridade, a efetivação dos direitos referentes à vida, à saúde, à alimentação, à educação, ao esporte, ao lazer, à profissionalização, à cultura, à dignidade, ao respeito, à liberdade e à convivência familiar e comunitária.

Em conjunto, o ECA, o SUS e a Reforma Psiquiátrica formam o arcabouço central das políticas públicas 
voltadas à saúde mental de crianças e adolescentes, tendo a intersetorialidade como fundamento principal das ações de cuidado. A intersetorialidade implica trabalho articulado e integrador de sujeitos, saberes, práticas e setores da sociedade, em especial a educação, a assistência social, a cultura, o lazer e a Justiça.

Desse modo, a intersetorialidade proporciona significativo aumento do potencial resolutivo no setor saúde e a permanente associação de diferentes sujeitos, saberes, práticas e setores disponibiliza uma rede de cuidados situados ao público infantojuvenil ${ }^{17}$. 0 trabalho intersetorial também propicia reflexão sobre as práticas sociais em construção, buscando novos modelos organizacionais que atendam às demandas de saúde da população em perspectiva humanizada e integral. Sob essa ótica, as políticas públicas de saúde mental se mostram capazes de contemplar os múltiplos aspectos do processo saúde-doença ${ }^{18}$.

0 primeiro resultado concreto advindo do reconhecimento da criança e do adolescente como sujeitos psíquicos singulares foi a criação, em 2002, dos CAPSi. Eles desenvolvem um trabalho em rede pautado na noção de clínica ampliada e na lógica da atenção diária a crianças e adolescentes com problemas de saúde mental. As ações são realizadas por equipes multiprofissionais com o propósito de oferecer acompanhamento clínico e promover a reinserção social, fortalecendo os vínculos familiares e comunitários de seus usuários ${ }^{19}$.

Segundo as cartilhas mais recentes do Ministério da Saúde, que tratam de orientações para a elaboração de projetos, além da reforma e ampliação dos CAPS e das unidades e atendimento, os CAPSi são indicados para regiões ou municípios com população acima de 70 mil habitantes. Para que suas ações obtenham resultados mais significativos, devem contar com uma equipe multidisciplinar mínima, composta por: 1 médico com formação em saúde mental (psiquiatra, neurologista ou pediatra); 1 enfermeiro; 4 profissionais com Ensino Superior (contemplando as categorias psicólogo, terapeuta ocupacional, fonoaudiólogo, assistente social, pedagogo ou outro profissional relevante para a configuração do projeto terapêutico singular); e 5 profissionais com Ensino Médio (contemplando as categorias técnico educacional, técnico administrativo, técnico e/ou auxiliar de enfermagem ou artesão) ${ }^{14}$.

A Rede de Atenção Psicossocial (RAPS) foi instituída no âmbito do SUS em 2011, com foco

\author{
...oferecer \\ acompanhamento \\ clínico e promover \\ a reinserção \\ social...
}

na criação, ampliação e articulação de pontos de atenção à saúde mental voltados a todas as pessoas que apresentam sofrimento ou transtorno psíquico e aquelas com necessidades decorrentes do uso de substâncias. De acordo com o Ministério da Saúde ${ }^{2}$, os objetivos específicos da RAPS são: a) promoção de cuidados à saúde voltados a grupos vulneráveis (crianças, adolescentes, jovens, pessoas em situação de rua e populações indígenas); b) prevenção do consumo e da dependência de crack, álcool e outras drogas; c) redução de danos provocados pelo consumo de crack, álcool e outras drogas; d) reabilitação e reinserção social de pessoas com transtorno mental e com necessidades decorrentes do uso de crack, álcool e outras drogas, por meio de acesso a trabalho, renda e moradia solidária; e e) melhoria da gestão dos serviços, parcerias intersetoriais etc. ${ }^{20}$.

No atual cenário das políticas públicas de saúde mental para crianças e adolescentes, um espaço de caráter tanto individual como coletivo, a psicologia se mostra cada vez mais necessária, com toda a sua cientificidade e sensibilidade para a abordagem da subjetividade humana. A atuação do psicólogo nesse contexto demanda uma nova práxis cotidiana que proporcione cuidado individualizado e humanizado aos usuários dos serviços, em linha com os princípios do SUS.

\section{Atuação do psicólogo em políticas públicas de saúde mental para crianças e adolescentes}

A presença do psicólogo no cenário das políticas públicas de saúde mental para crianças e adolescentes demanda um redirecionamento das práticas psicológicas, mostrando-se necessária uma reavaliação e adaptação dos modelos de atuação, de modo a proporcionar cuidado individualizado e humanizado aos usuários dos serviços. Estes devem assumir protagonismo em relação ao processo saúdedoença e as chamadas intervenções tradicionais, voltadas apenas à cura da doença, devem ser substituídas. A antiga visão individualista da 
psicologia, associada a uma atuação caracterizada como elitista na clínica privada, cede cada vez mais espaço a uma nova visão coletivista, em prol da dinâmica social e da promoção das subjetividades que se entrelaçam nas comunidades 21 .

Sabe-se que a representação social do psicólogo no âmbito das políticas públicas foi particularmente passível de equívocos no Brasil. Ao longo de nossa história, grande parte da população considerou o psicólogo um profissional inútil e classificou a psicologia como uma área irrelevante no setor saúde. Entretanto, nas últimas décadas, o senso comum vem mudando bastante ao observar a atuação do psicólogo nas comunidades. A aceitação desse profissional é cada vez maior no conjunto das práticas de atendimento aos diversos setores da sociedade, principalmente em termos de saúde mental e saúde coletiva, e diversas pesquisas com abordagens quantitativas e qualitativas demonstram a importância do psicólogo no tratamento de doenças de natureza psicológica ou no acompanhamento psicológico especializado. Hoje, os psicólogos que atuam na atenção à saúde mental para crianças e adolescentes têm direcionado seus esforços para ações articuladas com outros setores, trabalhando o bem-estar biopsicossocial, isto é, eles trabalham em rede nas novas matrizes do SUS e contam com apoio da comunidade e de seus inúmeros atores ${ }^{22}$.

No campo da saúde mental infantojuvenil, os psicólogos podem atuar em praticamente todos os componentes nos quais a RAPS 22 se encontra organizada, uma vez que ela reforça o cuidado a grupos vulneráveis e promove a integralidade da assistência preconizada pelo SUS. Tais componentes e seus pontos de atenção são ilustrados na Tabela 1.

Tabela 1. Componentes da Rede de Atenção Psicossocial no Brasil.

\begin{tabular}{ll}
\hline Componente & Pontos de atenção \\
\hline & Unidade básica de saúde \\
& Núcleo de apoio à saúde da família \\
Atenção básica à saúde & Consultório na Rua \\
& $\begin{array}{l}\text { Apoio aos serviços do componente atenção residencial de caráter } \\
\text { transitório }\end{array}$ \\
& Centros de convivência e cultura \\
Atenção psicossocial estratégica & Centro de Atenção Psicossocial, em suas diferentes modalidades
\end{tabular}

Samu 192

Sala de estabilização

Atenção de urgência e emergência UPA 24 horas

Portas hospitalares de atenção à urgência/pronto-socorro

Unidades básicas de saúde

\begin{tabular}{ll}
$\begin{array}{ll}\text { Atenção residencial de caráter } \\
\text { transitório }\end{array}$ & $\begin{array}{l}\text { Unidade de acolhimento } \\
\text { Serviço de atenção em regime residencial }\end{array}$ \\
$\begin{array}{ll}\text { Enfermaria especializada em regime residencial } \\
\text { Atenção hospitalar }\end{array}$ & $\begin{array}{l}\text { Serviço hospitalar de referência para atenção às pessoas com } \\
\text { sofrimento ou transtorno mental e com necessidades decorrentes } \\
\text { do uso de crack, álcool e outras drogas }\end{array}$ \\
\hline \multirow{2}{*}{ Estratégias de desinstitucionalização } & $\begin{array}{l}\text { Serviços residenciais terapêuticos } \\
\text { Programa de Volta para Casa }\end{array}$ \\
\hline \multirow{2}{*}{ Estratégias de reabilitação psicossocial } & $\begin{array}{l}\text { Iniciativas de geração de trabalho e renda } \\
\text { Empreendimentos solidários e cooperativas sociais }\end{array}$ \\
\hline
\end{tabular}

Fonte: Elaborada pelos autores.

Ao atuar nesses dispositivos, o psicólogo contribui de modo significativo tanto para o cidadão (em caráter individual), como para a sociedade (em caráter coletivo). As ações pautadas pela promoção da saúde e prevenção de doenças proporcionam suporte à atuação de outros profissionais comprometidos com o bem-estar da população, em especial das crianças e dos adolescentes, considerados seres humanos em desenvolvimento. Ao 
promover a saúde emocional e psíquica, o psicólogo potencializa as demais práticas de cuidado à saúde oferecidas à população.

Com tudo isso em vista, pode-se afirmar que a atuação do psicólogo no contexto das políticas públicas de saúde mental para crianças e adolescentes se mostra essencial para o desenvolvimento biopsicossocial de nossa sociedade como um todo, uma vez que esses sujeitos de direitos constroem e reconstroem suas realidades por meio de um processo dinâmico e contínuo.

\section{CONCLUSÃO}

No Brasil, as políticas públicas de saúde mental para crianças e adolescentes, mesmo sendo relativamente recentes, compreendem um vasto campo de aprendizagem e conhecimento. Apesar do progresso observado nos últimos anos, há inúmeros desafios a superar tanto em termos de atenção à saúde individual como coletiva, pois o público infantojuvenil demanda constante atualização e a criação de estratégias específicas de atuação que valorizem a troca de informações entre os profissionais da saúde que atendem seres humanos em desenvolvimento.

Uma limitação constatada neste artigo é a pequena quantidade de estudos sobre a atuação do psicólogo no âmbito das políticas de saúde mental para crianças e adolescentes. Há necessidade de novas pesquisas que investiguem a estruturação e a organização dessas políticas, bem como a relação entre o embasamento teórico e a prática profissional na área da psicologia, a fim de mensurar sua efetividade e eficácia diante das demandas envolvidas nas políticas públicas atuais.

Por fim, pode-se afirmar que este artigo aponta caminhos de pesquisa acerca da atuação do psicólogo, aprimorando o entendimento sobre a evolução do cuidado à saúde mental do público infantojuvenil. Tal iniciativa contribui para oferecer uma atenção psicológica cada vez mais individualizada e humanizada nos serviços de saúde, em linha com os princípios do SUS, com potenciais repercussões positivas na qualidade de vida da população brasileira.

\section{CONTRIBUIÇÃO DOS AUTORES}

Fabiana Cruz Soares contribuiu com 0 delineamento do estudo e a redação e revisão crítica

\author{
....o público \\ infantojuvenil demanda \\ constante atualização $e$ \\ a criação de estratégias \\ específicas de \\ atuação...
}

do manuscrito. Ramnsés Silva e Araújo e Renata Oliveira Sampaio Chaves contribuíram com a realização da pesquisa e a redação e revisão crítica do manuscrito.

\section{REFERÊNCIAS}

1. Couto M, Delgado P. Crianças e adolescentes na agenda política da saúde mental brasileira: inclusão tardia, desafios atuais. Psicol Clín [serial on the internet]. 2015 [cited 2017 Apr 28];27(1):1740. Available from: http://www.scielo.br/pdf/pc/ v27n1/0103-5665-pc-27-01-00017.pdf

2. Brasil. Atenção psicossocial a crianças e adolescentes no SUS: tecendo redes para garantir direitos. Brasília (DF): Ministério da Saúde; 2014.

3. Ribeiro EL, Paula CS. Políticas de saúde mental para crianças e adolescentes. In: Mateus MD, organizer. Políticas de saúde mental: baseado no curso de Políticas Públicas de Saúde Mental, do CAPS Luiz R. Cerqueira. São Paulo: Instituto de Saúde; 2013. p. 322-344.

4. Paes JPL. 0 Código de Menores e o Estatuto da Criança e do Adolescente: avanços e retrocessos [document on the internet]. 2013 [cited $2017 \mathrm{Apr}$ 29]. Available from: http://www.egov.ufsc.br/ portal/conteudo/o-c\%C3\%B3digo-de-menores-eo-estatuto-da-crian \%C $3 \%$ A 7a-e-do-adolescenteavan $\%$ C $3 \%$ A 7os-e-retrocessos

5. Fonseca JB. Código de Menores e o Estatuto da Criança e do Adolescente [document on the internet]. 2014 [cited 2017 Apr 29]. Available from: https://juliabr.jusbrasil.com.br/artigos/155146196/ codigo-de-menores-e-o-estatuto-da-crianca-e-doadolescente

6. Peroza MAR. Infância, educação e dignidade humana: considerações sobre os processos educativos da criança. Práxis Educativa [serial on the internet]. 2017 [cited 2017 May 22];13(1):1-19. Available from: http://www.revistas2.uepg.br/index.php/ praxiseducativa/article/view/10552/6-145 
7. Rossato LA, Lépore PE, Cunha RS. Estatuto da Criança e do Adolescente: comentado artigo por artigo: Lei 8.069/1990. São Paulo: Saraiva; 2012.

8. Gomes IS, Carminha IO. Guia para estudos de revisão sistemática: uma opção metodológica para as Ciências do Movimento Humano. Movimento (Porto Alegre) [serial on the internet]. 2014 [cited 2017 May 23];20(1):395-411. Available from: https:// seer.ufrgs.br/Movimento/article/view/41542/28358

9. Neves LMB, Jankoski AD, Schnaider MJ. Tutorial de pesquisa bibliográfica [document on the internet]. 2013 [cited 2017 May 24]. Available from: https:// portal.ufpr.br/pesquisa bibliogr bvs sd.pdf

10. Schraiber LB. Necessidades de saúde, políticas públicas e gênero: a perspectiva das práticas profissionais. Ciênc Saúde Colet [serial on the internet]. 2013 [cited 2017 May 25];17(10):263544. Available from: http://www.scielo.br/pdf/csc/ v17n10/13.pdf

11. Paiva CHA, Teixeira LA. Reforma Sanitária e a criação do Sistema Único de Saúde: notas sobre contextos e autores. Hist Ciênc Saúde - Manguinhos [serial on the internet]. 2014 [cited 2017 May 28];21(1):15-36. Available from: http://www.scielo. br/pdf/hcsm/v21n1/0104-5970-hcsm-21-1-00015.pdf

12. Trabuco KE0, Santos DS. Da Reforma Sanitária à Reforma Psiquiátrica: os movimentos sociais e a conquista de direitos. VII Jornada Internacional de Políticas Públicas; 2015; São Luís. Anais. São Luís: Universidade Federal do Maranhão; 2015.

13. Costa JP, Jorge MSB, Coutinho MPL, Costa EC, Holanda ÍTA. A Reforma Psiquiátrica e seus desdobramentos: representações sociais dos profissionais e usuários da atenção psicossocial. Psicol Saber Soc [serial on the internet]. 2016 [cited 2017 Jun 2];5(1):35-45. Available from: http://www.e-publicacoes.uerj.br/index.php/psisabersocial/article/view/15855/17903

14. Brasil. Centros de atenção psicossocial e unidades de acolhimento como lugares da atenção psicossocial nos territórios: orientações para elaboração de projetos de construção, reforma e ampliação de CAPS e de UA. Brasília (DF): Ministério da Saúde; 2015.

15. Melo AMC. Apontamentos sobre a Reforma Psiquiátrica no Brasil. Cadernos Brasileiros de Saúde Mental [serial on the internet]. 2012 [cited 2017 Jun 2];8(9):84-95. Available from: http:// incubadora.periodicos.ufsc.br/index.php/cbsm/ article/view/2127/2920

16. Silva ME. Histórico da saúde mental no Brasil até o governo atual. In: Castro CLF, Gontijo CRB, Almeida BV, organizers. Políticas públicas em saúde mental: abordagens e desafios. Belo Horizonte: Ed. UEMG; 2013. p. 18-63.
17. Nunes CK, Kantorski LP, Coimbra VCC. Interfaces entre serviços e ações da Rede de Atenção Psicossocial às crianças e adolescentes. Rev Gaúcha Enferm [serial on the internet]. 2016 [cited 2017 Jun 8];37(3):18. Available from: http://www.scielo.br/pdf/rgenf/ v37n3/0102-6933-rgenf-1983-144720160354858.pdf

18. Warschauer M, Carvalho YM. 0 conceito "intersetorialidade": contribuições ao debate a partir do Programa Lazer e Saúde da Prefeitura de Santo André/SP. Saúde Soc [serial on the internet]. 2014 [cited 2017 Jun 10];23(1):191-203. Available from: http://www.scielo.br/pdf/sausoc/v23n1/0104-1290sausoc-23-01-00191.pdf

19. Oliveira ACB, Miranda L. Práticas clínicas e o cuidado possivel no CAPSi: perspectivas de uma equipe interdisciplinar. Contextos Clín [serial on the internet]. 2015 [cited 2017 Jun 2];8(1):99112. Available from: http://pepsic.bvsalud.org/pdf/ cclin/v8n1/v8n1a11.pdf

20. Araújo GR, Silva LMN, Nascimento SA, Lima RRR. 0 real e o ideal em um serviço substitutivo de saúde mental: um relato de experiência. Sanare (Sobral, Online) [serial on the internet]. 2015 [cited 2017 Nov 7];14(1):69-75. Available from: https://sanare. emnuvens.com.br/sanare/article/view/611/328

21. Cimino APN, Leite DFCCS. Psicologia e saúde pública: cartografia das modalidades de prática psicológica nas policlínicas. Revista Psicologia e Saúde [serial on the internet]. 2016 [cited 2017 Nov 7];8(1):14-23. Available from: http://pepsic. bvsalud.org/pdf/rpsaude/v8n1/v8n1a03.pdf

22. Brasil. RAPS - Rede de Atenção Psicossocial [document on the internet]. 2014 [cited 2017 Jun 11]. Available from: http://portalsaude.saude.gov. br/index.php/o-minis-terio/prin-ipal/secretarias /803-sas-raiz/daet-raiz/saude-mental/L2-saudemental/12588-raps-rede-de-atencao-psicossocial
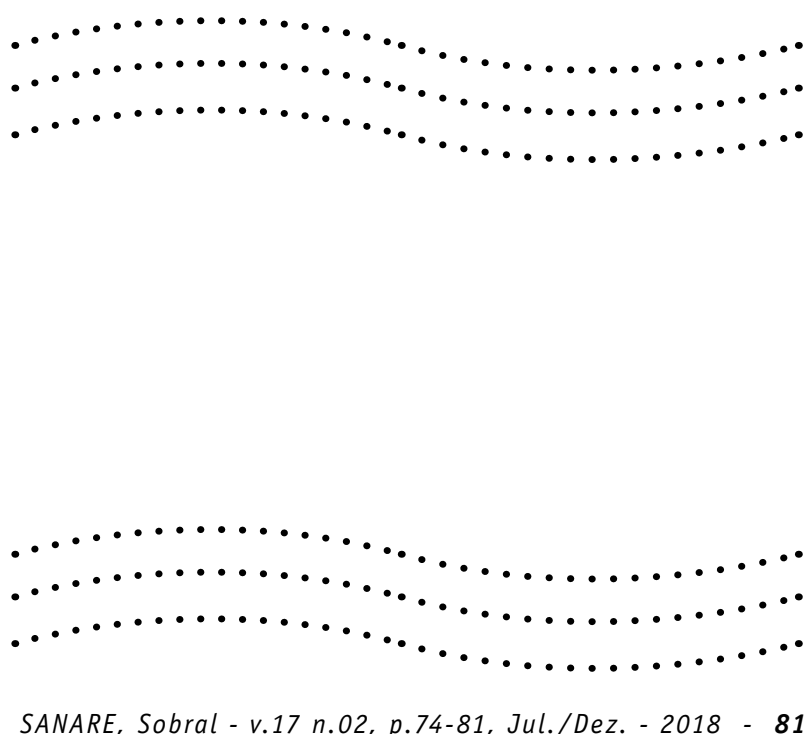